TAMKANG JOURNAL OF MATHEMATICS

Volume 33, Number 4, Winter 2002

\title{
ON THE BOUNDEDNESS OF A GENERALIZED FRACTIONAL INTEGRAL ON GENERALIZED MORREY SPACES
}

\author{
ERIDANI
}

\begin{abstract}
In this paper we extend Nakai's result on the boundedness of a generalized fractional integral operator from a generalized Morrey space to another generalized Morrey or Campanato space.
\end{abstract}

\section{Introduction and Main Results}

For a given function $\rho:(0, \infty) \rightarrow(0, \infty)$, let $\mathcal{T}_{\rho}$ be the generalized fractional integral operator, given by

$$
\mathcal{T}_{\rho} f(x)=\int_{\boldsymbol{R}^{n}} \frac{f(y) \rho(|x-y|)}{|x-y|^{n}} d y
$$

and put

$$
\tilde{\mathcal{T}}_{\rho} f(x)=\int_{\boldsymbol{R}^{n}} f(y)\left(\frac{\rho(|x-y|)}{|x-y|^{n}}-\frac{\rho(|y|)\left(1-\chi_{B_{0}}(y)\right)}{|y|^{n}}\right) d y,
$$

the modified version of $\mathcal{T}_{\rho}$, where $B_{0}$ is the unit ball about the origin, and $\chi_{B_{0}}$ is the characteristic function of $B_{0}$.

In [4], Nakai proved the boundedness of the operators $\tilde{\mathcal{T}}_{\rho}$ and $\mathcal{T}_{\rho}$ from a generalized Morrey space $\mathcal{M}_{1,{ }_{\phi}}$ to another generalized Morrey space $\mathcal{M}_{1},{ }_{\psi}$ or generalized Campanato space $\mathcal{L}_{1}, \psi$. More precisely, he proved that

$$
\left\|\mathcal{T}_{\rho} f\right\|_{\mathcal{M}_{1}, \psi} \leq C\|f\|_{\mathcal{M}_{1}, \phi} \text { and }\left\|\tilde{\mathcal{T}}_{\rho} f\right\|_{\mathcal{L}_{1}, \psi} \leq C\|f\|_{\mathcal{M}_{1}, \phi},
$$

where $C>0$, with some appropriate conditions on $\rho, \phi$ and $\psi$. Using the techniques developed by Kurata et.al.[1], we investigate the boundedness of these operators from generalized Morrey spaces $\mathcal{M}_{p, \phi}$ to generalized Morrey spaces $\mathcal{M}_{p}, \psi$ or generalized Campanato spaces $\mathcal{L}_{p}, \psi$ for $1<p<\infty$.

The generalized Morrey and Campanato spaces are defined as follows. For a given function $\phi:(0, \infty) \rightarrow(0, \infty)$, and $1<p<\infty$, let

$$
\|f\|_{\mathcal{M}_{p, \phi}}=\sup _{B} \frac{1}{\phi(B)}\left(\frac{1}{|B|} \int_{B}|f(y)|^{p} d y\right)^{\frac{1}{p}}
$$

Received September 19, 2001; revised January 29, 2002.

2000 Mathematics Subject Classification. Primary 42B20, 46E30, 43A15, 26A33.

Key words and phrases. Generalized fractional integrals, generalized Morrey spaces, generalized Campanato spaces. 
and

$$
\|f\|_{\mathcal{L}_{p, \phi}}=\sup _{B} \frac{1}{\phi(B)}\left(\frac{1}{|B|} \int_{B}\left|f(y)-f_{B}\right|^{p} d y\right)^{\frac{1}{p}},
$$

where the supremum is taken over all open balls $B=B(a, r)$ in $\boldsymbol{R}^{n},|B|$ is the Lebesgue measure of $B$ in $\boldsymbol{R}^{n}, \phi(B)=\phi(r)$, and $f_{B}=\frac{1}{|B|} \int_{B} f(y) d y$. We define the generalized Morrey space $\mathcal{M}_{p, \phi}$ by

$$
\mathcal{M}_{p, \phi}=\left\{f \in L_{l o c}^{p}\left(\boldsymbol{R}^{n}\right):\|f\|_{\mathcal{M}_{p, \phi}}<\infty\right\},
$$

and the generalized Campanato space $\mathcal{L}_{p, \psi}$ by

$$
\mathcal{L}_{p, \phi}=\left\{f \in L_{l o c}^{p}\left(\boldsymbol{R}^{n}\right):\|f\|_{\mathcal{L}_{p, \phi}}<\infty\right\} .
$$

Our results are the following:

Theorem 1. If $\rho, \phi, \psi:(0, \infty) \longrightarrow(0, \infty)$ satisfying the conditions below:

$$
\begin{aligned}
& \frac{1}{2} \leq \frac{t}{r} \leq 2 \Rightarrow \frac{1}{A_{1}} \leq \frac{\phi(t)}{\phi(r)} \leq A_{1}, \text { and } \frac{1}{A_{2}} \leq \frac{\rho(t)}{\rho(r)} \leq A_{2}, \\
& \int_{0}^{1} \frac{\rho(t)}{t} d t<\infty, \text { and for all } r>0, \text { we have } \int_{r}^{\infty} \frac{\phi(t)^{p}}{t} d t \leq A_{3} \phi(r)^{p}, \\
& \phi(r) \int_{0}^{r} \frac{\rho(t)}{t} d t+\int_{r}^{\infty} \frac{\rho(t) \phi(t)}{t} d t \leq A_{4} \psi(r), \text { for all } r>0,
\end{aligned}
$$

where $A_{i}>0$ are independent of $t, r>0$, then for each $1<p<\infty$ there exists $C_{p}>0$ such that

$$
\left\|\mathcal{T}_{\rho} f\right\|_{\mathcal{M}_{p, \psi}} \leq C_{p}\|f\|_{\mathcal{M}_{p, \phi}} .
$$

Theorem 2. If $\rho, \phi, \psi:(0, \infty) \rightarrow(0, \infty)$ satisfying the conditions below:

$$
\begin{aligned}
& \frac{1}{2} \leq \frac{t}{r} \leq 2 \Rightarrow \frac{1}{A_{1}} \leq \frac{\phi(t)}{\phi(r)} \leq A_{1}, \text { and } \frac{1}{A_{2}} \leq \frac{\rho(t)}{\rho(r)} \leq A_{2}, \\
& \int_{0}^{1} \frac{\rho(t)}{t} d t<\infty, \text { and for all } r>0, \text { we have } \int_{r}^{\infty} \frac{\phi(t)^{p}}{t} d t \leq A_{3} \phi(r)^{p}, \\
& \left|\frac{\rho(r)}{r^{n}}-\frac{\rho(t)}{t^{n}}\right| \leq A_{4}|r-t| \frac{\rho(r)}{r^{n+1}}, \text { for } \frac{1}{2} \leq \frac{t}{r} \leq 2, \\
& \phi(r) \int_{0}^{r} \frac{\rho(t)}{t} d t+r \int_{r}^{\infty} \frac{\rho(t) \phi(t)}{t^{2}} d t \leq A_{5} \psi(r), \text { for all } r>0,
\end{aligned}
$$

where $A_{i}>0$ are independent of $t, r>0$, then for each $1<p<\infty$ there exists $C_{p}>0$ such that

$$
\left\|\tilde{\mathcal{T}}_{\rho} f\right\|_{\mathcal{L}_{p, \psi}} \leq C_{p}\|f\|_{\mathcal{M}_{p, \phi}} .
$$




\section{Proof of the Theorems}

To prove the theorems, we shall use the following result of Nakai [2] (in a slightly modified version) about the boundedness of the standard maximal function $M f$ on a generalized Morrey space $\mathcal{M}_{p, \phi}$. The standard maximal function $M f$ is defined by

$$
M f(x)=\sup _{B \ni x} \frac{1}{|B|} \int_{B}|f(y)| d y, x \in \boldsymbol{R}^{n},
$$

where the supremum is taken over all open balls $B$ containing $x$.

Theorem(Nakai). If $\phi:(0, \infty) \longrightarrow(0, \infty)$ satisfying the conditions below:

(a) $\frac{1}{2} \leq \frac{t}{r} \leq 2 \Rightarrow \frac{1}{A_{1}} \leq \frac{\phi(t)}{\phi(r)} \leq A_{1}$,

(b) $\int_{r}^{\infty} \frac{\phi(t)^{p}}{t} d t \leq A_{2} \phi(r)^{p}$, for all $r>0$,

where $A_{i}>0$ are independent of $t, r>0$, then for each $1<p<\infty$ there exists $C_{p}>0$ such that

$$
\|M f\|_{\mathcal{M}_{p, \phi}} \leq C_{p}\|f\|_{\mathcal{M}_{p, \phi}} .
$$

From now on, $C$ and $C_{p}$ will denote positive constants, which may vary from line to line. In general, these constants depend on $n$.

Proof of Theorem 1. For $x \in \boldsymbol{R}^{n}$, and $r>0$, write

$$
\mathcal{T}_{\rho} f(x)=\int_{|x-y|<r} \frac{f(y) \rho(|x-y|)}{|x-y|^{n}} d y+\int_{|x-y| \geq r} \frac{f(y) \rho(|x-y|)}{|x-y|^{n}} d y=I_{1}(x)+I_{2}(x) .
$$

Note that, for $t \in\left[2^{k} r, 2^{k+1} r\right]$, there exist constants $C_{i}>0$ such that

$$
\rho\left(2^{k} r\right) \leq C_{1} \int_{2^{k} r}^{2^{k+1} r} \frac{\rho(t)}{t} d t
$$

and

$$
\rho\left(2^{k} r\right) \phi\left(2^{k} r\right) \leq C_{2} \int_{2^{k} r}^{2^{k+1} r} \frac{\rho(t) \phi(t)}{t} d t .
$$

So, we have

$$
\begin{aligned}
\left|I_{1}(x)\right| & \leq \int_{|x-y|<r} \frac{|f(y)| \rho(|x-y|)}{|x-y|^{n}} d y \\
& \leq \sum_{k=-\infty}^{-1} \int_{2^{k} r \leq|x-y|<2^{k+1} r} \frac{|f(y)| \rho(|x-y|)}{|x-y|^{n}} d y \\
& \leq C \sum_{k=-\infty}^{-1} \frac{\rho\left(2^{k} r\right)}{\left(2^{k} r\right)^{n}} \int_{|x-y|<2^{k+1} r}|f(y)| d y
\end{aligned}
$$




$$
\begin{aligned}
& \leq C \sum_{k=-\infty}^{-1} \rho\left(2^{k} r\right) M f(x) \\
& \leq C M f(x) \sum_{k=-\infty}^{-1} \int_{2^{k} r}^{2^{k+1 r}} \frac{\rho(t)}{t} d y \\
& \leq C M f(x) \int_{0}^{r} \frac{\rho(t)}{t} d y \\
& \leq C \frac{\psi(r)}{\phi(r)} M f(x) .
\end{aligned}
$$

Meanwhile,

$$
\begin{aligned}
\left|I_{2}(x)\right| & \leq \int_{|x-y| \geq r} \frac{|f(y)| \rho(|x-y|)}{|x-y|^{n}} d y \\
& \leq \sum_{k=0}^{\infty} \int_{2^{k} r \leq|x-y|<2^{k+1} r} \frac{|f(y)| \rho(|x-y|)}{|x-y|^{n}} d y \\
& \leq C \sum_{k=0}^{\infty} \frac{\rho\left(2^{k+1} r\right)}{\left(2^{k} r\right)^{n}} \int_{|x-y|<2^{k+1} r}|f(y)| d y \\
& \leq C \sum_{k=0}^{\infty} \rho\left(2^{k+1} r\right) \phi\left(2^{k+1} r\right)\|f\|_{\mathcal{M}_{p, \phi}} \\
& \leq C\|f\|_{\mathcal{M}_{p, \phi}} \sum_{k=0}^{\infty} \int_{2^{k+1} r}^{2^{k+2} r} \frac{\phi(t) \rho(t)}{t} d t \\
& \leq C\|f\|_{\mathcal{M}_{p, \phi}} \int_{r}^{\infty} \frac{\phi(t) \rho(t)}{t} d t \\
& \leq C \psi(r)\|f\|_{\mathcal{M}_{p, \phi}} .
\end{aligned}
$$

Now, for $1 \leq p<\infty$, we have

$$
\left|\mathcal{T}_{\rho} f(x)\right|^{p} \leq 2^{p-1}\left(\left|I_{1}(x)\right|^{p}+\left|I_{2}(x)\right|^{p}\right),
$$

and by Nakai's Theorem, we have for all balls $B=B(a, r)$

$$
\frac{1}{\psi(r)^{p}|B|} \int_{B}\left|I_{1}(x)\right|^{p} d x \leq \frac{C}{\phi(r)^{p}|B|} \int_{B} M f(x)^{p} d x \leq C\|M f\|_{\mathcal{M}_{p, \phi}}^{p} \leq C_{p}\|f\|_{\mathcal{M}_{p, \phi}}^{p},
$$

and

$$
\frac{1}{\psi(r)^{p}|B|} \int_{B}\left|I_{2}(x)\right|^{p} d x \leq C\|f\|_{\mathcal{M}_{p, \phi}}^{p} .
$$

Combining the two estimates, we obtain

$$
\frac{1}{\psi(r)^{p}|B|} \int_{B}\left|\mathcal{T}_{\rho} f(x)\right|^{p} d x \leq C_{p}\|f\|_{\mathcal{M}_{p, \phi}}^{p},
$$


and the result follows.

Proof of Theorem 2. Let $\tilde{B}=B(a, 2 r)$. For $x \in B=B(a, r)$, we have

$$
\tilde{\mathcal{T}}_{\rho} f(x)-C_{B}=E_{B}^{1}(x)+E_{B}^{2}(x),
$$

where

and

$$
\begin{aligned}
C_{B} & =\int_{\boldsymbol{R}^{n}} f(y)\left(\frac{\rho(|a-y|)\left(1-\chi_{\tilde{B}}(y)\right)}{|a-y|^{n}}-\frac{\rho(|y|)\left(1-\chi_{B_{0}}(y)\right)}{|y|^{n}}\right) d y, \\
E_{B}^{1}(x) & =\int_{\tilde{B}} f(y) \frac{\rho(|x-y|)}{|x-y|^{n}} d y,
\end{aligned}
$$

$$
E_{B}^{2}(x)=\int_{\tilde{B}^{c}} f(y)\left(\frac{\rho(|x-y|)}{|x-y|^{n}}-\frac{\rho(|a-y|)}{|a-y|^{n}}\right) d y .
$$

From (2.3), we have

$$
\left|C_{B}\right| \leq C\left(\int_{|a-y|<k}|f(y)| d y+|a| \int_{|a-y| \geq k}|f(y)| \frac{\rho(|a-y|)}{|a-y|^{n+1}} d y\right),
$$

where $k=\max (2|a|, 2 r)$, and so we know that $C_{B}$ is finite for every ball $B=B(a, r)$.

With the same technique as in the proof of the previous theorem, we have

$$
\begin{aligned}
\left|E_{B}^{1}(x)\right| & \leq \int_{|a-y|<2 r} \frac{|f(y)| \rho(|x-y|)}{|x-y|^{n}} d y \\
& \leq \int_{|x-y|<3 r} \frac{|f(y)| \rho(|x-y|)}{|x-y|^{n}} d y \\
& \leq C M f(x) \int_{0}^{3 r} \frac{\rho(t)}{t} d t \\
& \leq C M f(x) \int_{0}^{r} \frac{\rho(t)}{t} d t,
\end{aligned}
$$

and by $(2.3)$

$$
\begin{aligned}
\left|E_{B}^{2}(x)\right| & \leq \int_{|a-y| \geq 2 r}|f(y)|\left|\frac{\rho(|x-y|)}{|x-y|^{n}}-\frac{\rho(|a-y|)}{|a-y|^{n}}\right| d y \\
& \leq C|x-a| \int_{|a-y| \geq 2 r}|f(y)| \frac{\rho(|a-y|)}{|a-y|^{n+1}} d y \\
& \leq C|| f \|_{\mathcal{M}_{p, \phi}} r \int_{r}^{\infty} \frac{\rho(t) \phi(t)}{t^{2}} d t,
\end{aligned}
$$

and the result follows as before.

Remark. We also suspect that $\tilde{\mathcal{T}}_{\rho}$, the modified version of $\mathcal{T}_{\rho}$, is bounded from $\mathcal{L}_{p, \phi}$ to $\mathcal{L}_{p, \psi}$ under the same hypothesis on $\rho, \phi$ and $\psi$ as in Theorem 2. However, we have not obtained the proof and the research in this direction is still ongoing. 


\section{Acknowledgements}

The author would like to thank Dr. Hendra Gunawan and Dr. Eiichi Nakai for their useful comments and suggestions during the preparation of this note. He also thanks the referee(s) for their remarks on the earlier version of this note.

\section{References}

[1] K. Kurata, S. Nishigaki and S. Sugano, Boundedness of integral operators on generalized Morrey spaces and its application to Schrödinger operators, Proc. Amer. Math. Soc. 128(1999), 1125-1134.

[2] E. Nakai, Hardy-Littlewood maximal operator, singular integral operators, and the Riesz potentials on generalized Morrey spaces, Math. Nachr. 166(1994), 95-103.

[3] E. Nakai, On generalized fractional integrals, Proceedings of the International Conference on Mathematical Analysis and its Applications 2000 (Kaohsiung, Taiwan), Taiwanese J. Math. 5(2001), 587-602.

[4] E. Nakai, On generalized fractional integrals on the weak Orlicz spaces, $B M O_{\phi}$, the Morrey sapces and the Campanato spaces, Proceedings of the Conference on Function Spaces, Interpolation Theory and related topics in honour of Jaak Peetre on his 65th birthday, Lund University Sweden, to appear.

Department of Mathematics, Bandung Institute of Technology, Bandung 40132, Indonesia.

Permanent address: Department of Mathematics, University of Airlangga, Surabaya 60115, Indonesia. 